\title{
Recognition and Real-Time Detection of Blinking Eyes on Electroencephalographic Signals Using Wavelet Transform
}

\author{
Renato Salinas ${ }^{1}$, Enzo Schachter ${ }^{1}$, and Michael Miranda ${ }^{2}$ \\ ${ }^{1}$ Departamento de Ingeniería Mecánica \\ ${ }^{2}$ Programa de Doctorado en Automatización \\ Facultad de Ingeniería, Universidad de Santiago de Chile, Santiago, Chile \\ \{renato.salinas, enzo.schachter, michael.mirandas\} @usach.cl
}

\begin{abstract}
In this paper we study the detection of a specific pattern associated with the blinking of an eye in real time using electroencephalogram (EEG) signals of a single channel. This paper takes into account the theoretical and practical principles enabling the design and implementation of a system for real-time detection of time location, regardless of scale and multiple incidences. By using wavelet transform it permits us the fulfillment of our objective. The multiple detection and real-time operation is achieved by working with a popup window giving the projection of an ongoing analysis of the signal sampled by the EEG.
\end{abstract}

Keywords: biological signals, electroencephalogram EEG, brain computer interface BCI, eye blink detection, pattern recognition, wavelet transform.

\section{Introduction}

The electroencephalogram or EEG was first used in humans by Hans Berger in 1924 [1], with the purpose of recording electric potentials of the brain. These signals are acquired from sensors called electrodes attached to the scalp of the subject. The function of an electrode is to passively collect electrical potentials from neuron banks that are located mainly in the cerebral cortex. The level of these signals is typically within the range of 40 to 100 microvolts [2]. Given their low electrical levels, EEG signals can be easily contaminated by other sources. An EEG signal that does not originate in the brain is called an artifact. The artifacts fall into two categories: physiological and non-physiological. Any source in the body that has an electric dipole generates an electric field capable of producing physiological artifacts. The non-physiological artifacts are produced by electrical and mechanical devices [3]. The human eye, similar to an electrical system, acts as a dipole with a positive charge in front and a negative charge in the back; the exercise of closing and opening the eyes produces artifacts of EEG signals [4]. Given that the artifacts are usually considered an unwanted signal or signal interference, this work is focused on real-time detection of a specific pattern generated by the blink of an eye so that they can be removed. The artifact generated by the blink of an eye is not necessarily a problem, but an opportunity, because its 
one-dimensional representation on a graph of microvolts vs. time is specific and independent of the individual from which the EEG signals are obtained [4]. Therefore, the artifact represents a clear identifiable pattern that can be used in brain computer interfaces -BCI-, if the artifacts are generated voluntarily by the user it can translate simple commands to help people with disabilities. Currently, there are numerous researches aimed at finding processing algorithms and classification of EEG patterns that will produce better results in the BCI [5] [6] [7]. At first glance, you may assume that having a specific pattern makes it easier to detect the presence of artifacts produced by the blinking of the eyes, but in practice it is not, because the pattern is presented at different scales and in multiple instances. In order for the detection algorithm to be robust it is necessary to consider an invariant model both in scale, translation and instances in which the pattern appears in the window of study, all in real time. This study takes advantage of the non-stationary signal analysis capabilities of the wavelet transform that, unlike the Fourier transform, it is a simultaneous representation of the time and the frequency domains [8].

\section{Acquisition and Processing of the EEG Signal}

\subsection{Signal Acquisition and Signal Transmission}

Human electroencephalography signals are typically below 256 Hz [9] (fmax), by the foregoing and in consideration of the Nyquist-Shannon sampling theorem, it is necessary to use a sampling frequency equal to or greater than twice the maximum frequency, i.e., at least $512 \mathrm{~Hz}$. For a practical reason we preferred to use dry contact sensors or active electrodes, which have an electrical microcircuit in the electrode to improve the quality of the acquired signal.
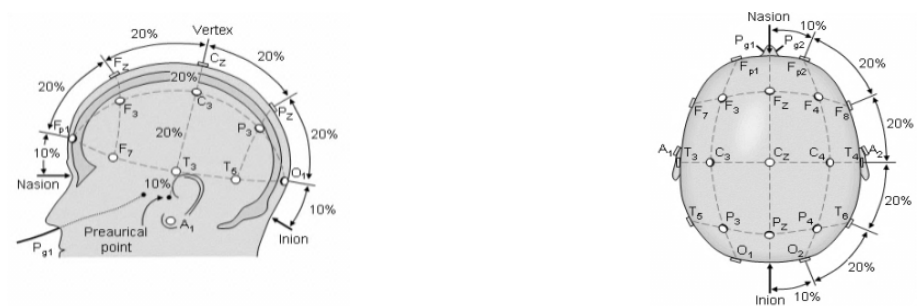

Fig. 1. The international 10-20 system. $\mathrm{A}=$ Ear lobe, $\mathrm{C}=$ central, $\mathrm{Pg}=$ nasopharyngeal, $\mathrm{P}=$ parietal, $\mathrm{F}=$ frontal, $\mathrm{Fp}=$ frontal polar, $\mathrm{O}=$ occipital. [9].

In the study of EEG signals, the cerebral cortex is divided into different zones; of which the nomenclature used depends on the system [10] [11]; in the case of the international system our goal of study focuses on the polar frontal areas FP1 and FP2 (Fig. 1). For the development of this work we used the Mindset of Neurosky Inc. This unit has only one dry contact EEG electrode that is located in the FP1, its frequency is $512 \mathrm{~Hz}$, and it includes a rechargeable battery, as well as a blue-tooth transmitter that sends the signal to the computer wirelessly. 


\subsection{EEG Signal Processing}

Upon receiving the signal on the computer, we construct a continuous recording of EEG data at 512 samples per second. The samples are extracted, one at a time from this record to build the analysis segment in the form of a moving window of $500[\mathrm{~ms}]$, i.e. a vector of 256 samples or components that are being renewed by entering a new sample. A sample data flow is shown in Fig 2.

$$
\cdots \rightarrow s^{n+1} \rightarrow\left[s_{256}^{n} \rightarrow s_{255}^{n-1} \rightarrow \cdots \rightarrow s_{2}^{n-2} \rightarrow s_{1}^{n-255}\right] \rightarrow s^{n-256} \rightarrow \cdots
$$

Fig. 2. A schematic example of the data flow within the analysis window of 256 components. The subscript represents the component in the analysis window; the superscript represents the number of signal sample under study.

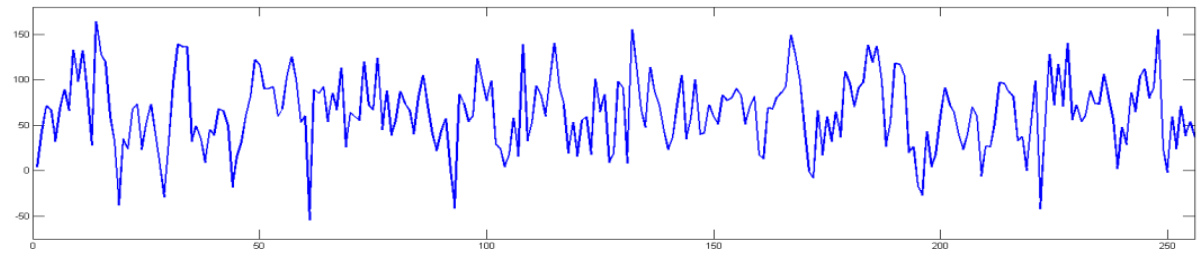

Fig. 3. Typical EEG signal without the presence of artifacts, captured by the processing system in an analysis window of 256 components, equal to $500 \mathrm{~ms}$

The number of components of the window was selected given the duration of a blink of an eye [12]. An example of EEG signal without artifacts is shown in Fig. 3. The moving window is processed with the low pass filter.

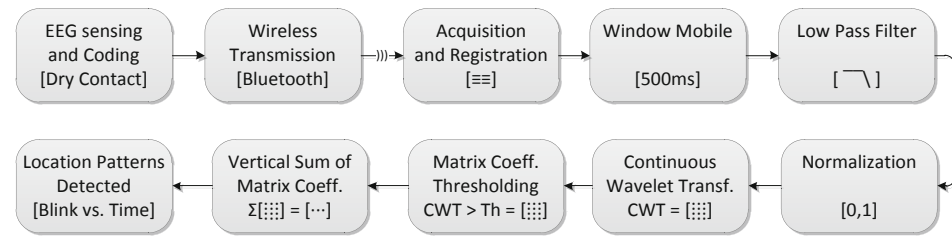

Fig. 4. Overall scheme of the recognition system and real-time detection of eye blinking in electroencephalographic EEG signals using wavelet transform

The step of acquiring and processing data in a global context is presented in the first row of the scheme depicted in Fig. 4. Once processed the analysis of the signal segment, the algorithm proceeds to the next step of detecting the eye-blinking pattern shown in the second row of the scheme of Fig. 4, the detection block yields the temporal location of each artifact caused by the blinking of the eyes.

\section{Characteristics and Effects of Eye Blinking in EEG Signals}

The artifact in the EEG signal produced by the blinking of an eye has a positive maxi-mum and a negative minimum, associated with eye opening and eye closing, 
respectively. In this paper we use a feature associated with shape that has low symmetry and a variable amplitude, duration and frequency, as shown in Fig. 5.

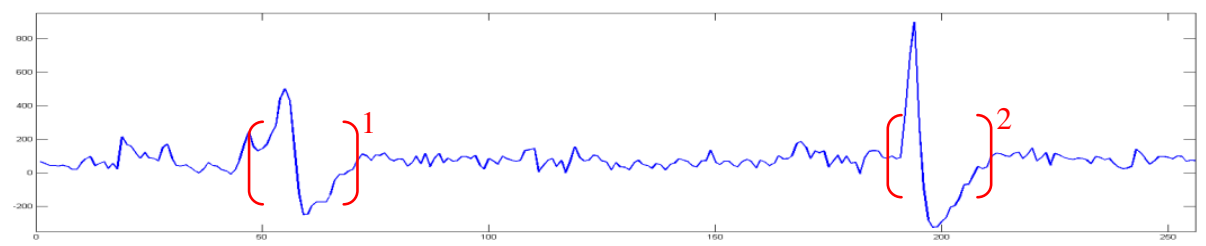

Fig. 5. EEG signal with two artifacts produced by blinking eyes. The artifacts are shown enclosed in square brackets.

Determining a unique shape pattern for the recursive detection of eye blinking present in a stochastic signal is useless, since it would only apply to a group of shapes that present some similarity to the chosen pattern. To improve the condition one may increase the number of different patterns in amplitude and duration, and then perform a recursive search, but it would consume enough time for each process cycle to damage the final condition of real-time analysis. Requiring a group of interrelated patterns is not entirely negative, as one might think of a family of patterns derived from a "mother" pattern that passes down the characteristics to the children patterns, to perform multi-resolution analysis. In our case, this paper deals with the analysis using wavelet transform.

\section{$4 \quad$ Wavelet Transform}

The analysis of signals with stationary components highlights the Fourier Transform FT, thanks to the ability to deliver a representation of frequency content present in the signal. However, it is not very useful for analyzing signals that do not possess stationary behavior, or those which have abrupt changes in very small intervals.

$\begin{aligned} & \text { Short Time FT } \\ & (\text { STFT })\end{aligned} \quad \operatorname{STFT}(f)=\int_{-\infty}^{\infty} x(t) g^{*}(t-b) e^{-i 2 \pi f t} d t$

To counteract the disadvantages there is an adapted version of the Fourier Transform called Short Time Fourier Transform STFT (Eq. 3), also known as Gabor Transform. In the equation 1 is given a function $g(t)$ used for analysis window moving over the data to determine the spectrum locally, variable $b$ indicates the position in which the analysis is made and $*$ denotes the complex conjugate. The disadvantage of this transform is that the analysis window is of a fixed size and therefore cannot adapt to the characteristics of the signal at certain points of interest to analyze.

Analysis by Wavelet Transform is a logical step following the STFT, using windows regions of variable sizes. Wavelet analysis allows the use of large time intervals in those segments where greater accuracy is required at low frequencies and smaller regions where information is required at high frequency. A wavelet is a waveform of limited duration that has average value zero. Wavelet analysis decomposes the signal into shifted and scaled versions of another signal called mother wavelet [13]. The 
Wavelet has a limited duration, it also tends to be irregular and asymmetric, allowing for greater adaptability and better convergence; for this reason, it is important to properly select the mother wavelet depending on the signal analyzed. There are two classes of wavelets with applications in different domains. The continuous wavelet transform and the discrete wavelet; these classes determine their particular properties. The discrete wavelet transform or DWT arises from a discretization in time and scale, unlike the continuous wavelet transform or CWT, which is used in this work.

\subsection{Continuous Wavelet Transform}

The continuous wavelet transform can be defined as the integral in the entire interval of time $t$ of the signal $x(t)$ multiplied by the scale of a shifted version of the mother function $\Psi(t)$ (equation 2).

Mother Wavelet

$$
\Psi_{a, b}(t)=\frac{1}{\sqrt{a}} \Psi\left(\frac{t-b}{a}\right) \quad(a, b) \in R, a \neq 0 .
$$

Continuous Wavelet

Transform (CWT)

$$
C W T(a, b)=\frac{1}{\sqrt{a}} \int_{-\infty}^{\infty} x(t) \Psi\left(\frac{t-b}{a}\right) d t
$$

The CWT (equation 3) delivers as a result coefficients depending on the scale $a$ and position $b$ of the mother wavelet generating a family of wavelets from the original, which is scaled and translated in each of these two subscripts, respectively. The application of these operations on this function generates the different areas of detail.

The main families of wavelets are Haar, Daubechies, Biorthogonal (bior), Coiflet, Morlet, Mexican Hut, Meyer and Symlet [14]. All have a specific equation that shows their differences in shape, as shown in Fig 6.

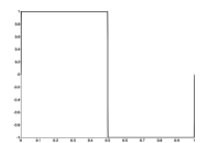

a) Haar

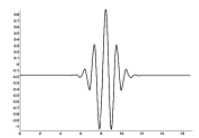

e) Morlet

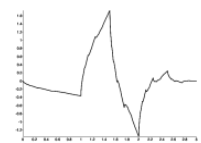

b) Daubechies

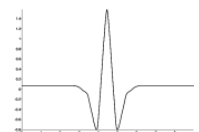

f) Mexican Hut

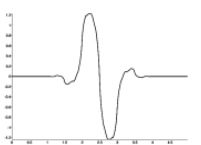

c) Bior 1.3

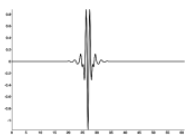

g) Meyer

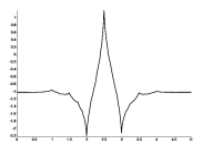

d) Coiflet

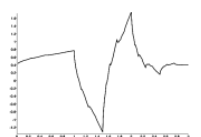

h) Symlet

Fig. 6. Typical shapes of the main wavelet families. (Source: Wavelet Toolbox Matlab®).

\section{Characteristic Pattern Detection}

Given the waveform pattern associated with eye blinking shown in Fig. 5, it is simple to find similarities with the Biorthogonal wavelet family, specifically bior1.3 (see Fig. $6 \mathrm{c}$ and Fig. 7); the condition of similarity is sufficient to use this wavelet in the analysis because it is a variant pattern which has different versions of scale and translation, which is a problem to be solved using the wavelet transform analysis. As seen in 
Fig. 4 the algorithm comprises a detection step which starts with the normalization of the analyzed signal, then bior 1.3 continuous wavelet transform is applied to obtain the coefficients which will be subsequently processed to obtain the location of each artifact. Using the signal shown in Fig. 5 and applying the continuous wavelets transform, we obtain a coefficient matrix, equation 4 . The dimensions of the matrix depend on the number of components or samples of the analyzed signal and the level or number of scales in the decomposition by the wavelet transform.

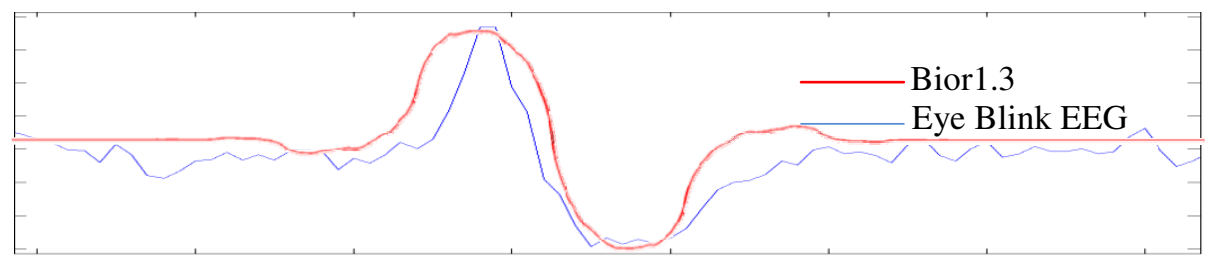

Fig. 7. Overlay of the artifact produced by eye blinking and the wavelet bior1.3

$$
\mathrm{C}(\mathrm{a}, \mathrm{b})=\mathrm{CWT} \text { ('input signal', 'bior1.3') }
$$

where $\mathrm{C}(\mathrm{a}, \mathrm{b})$ is the coefficients matrix and CWT is the continuous wavelet transform, applied to input signal.

This matrix can be plotted as if it were a gray scale image, with this you get the graph scale vs. time, i.e. coefficients $a$ vs. $b$ respectively obtained from equation 4 , see Fig. 8 , in which white vertical lines indicate the level of agreement of the wavelet used for signal analysis and study, like the images in grayscale, the higher-value components tend to be represented with a color ever closer to white [15]. In Fig. 8(a) there is a dark horizontal band corresponding to the first wavelet decomposition level, i.e., the high frequency; therefore, we can deduce that the searched pattern has no presence in a high frequency band. As you would in a grayscale image analysis [15], it is possible to apply thresholding to the matrix of wavelet coefficients, as defined in equation 5 .

$$
T(a, b)=\left\{\begin{array}{ccc}
0 & \text { if } & C(a, b)<\text { Threshold }_{\text {fixed }} \\
C(a, b) & , & \text { otherwise }
\end{array}\right.
$$

Fig. 8(b) shows the presence of two white vertical lines that represent the detection of the searched pattern and its location in time. The aim of this work is to automate the detection and do it in real time, so that speed of each process is very important.

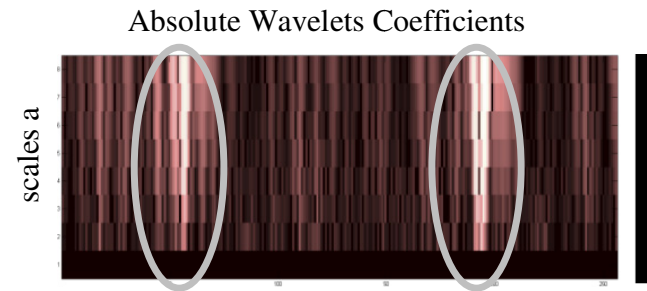

(a)
Binarized Wavelets Coefficients

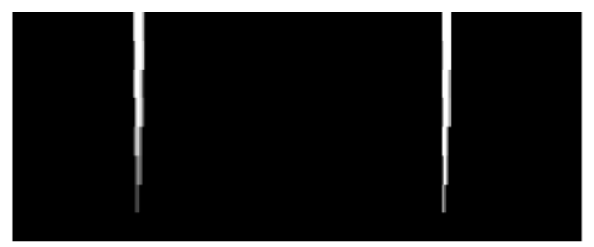

(b)

Fig. 8. (a) Applications of the continuous wavelet transform bior 1.3 to the signal shown in Fig. 5. (b) Binarization of the wavelet coefficient matrix using thresholding. 
In the case under study for this work the number of components or the analysis window size is of 256 samples and 8 levels of decomposition, which involves obtaining a coefficient matrix [C] of 8x256 elements. Any way you can use parameters other than those indicated for detection, which do not have a strong impact on the algorithm for the next step, reduces the number of rows in the matrix to just one row. To perform the dimensional reduction, the columns of the coefficient matrix $T(a, b)$ are added as shown in equation 6 . The vector $\mathrm{V}$ obtained in equation 6 gives us the actual location in time of the artifact produced by the eye blinking. Since the analysis is in real time, the data was smoothed with a $10 \mathrm{~ms}$. moving average.

$$
V(1,2, \ldots, n)=\sum_{a} T(a, b), \quad b=1,2, \ldots, n
$$

Applying the identification process to the signal shown in Fig. 5 results in the signal shown in Fig. 9, which overlap the input signal or analysis signal with the filtered output signal along with the location of artifacts detected produced by the blinking of an eye.

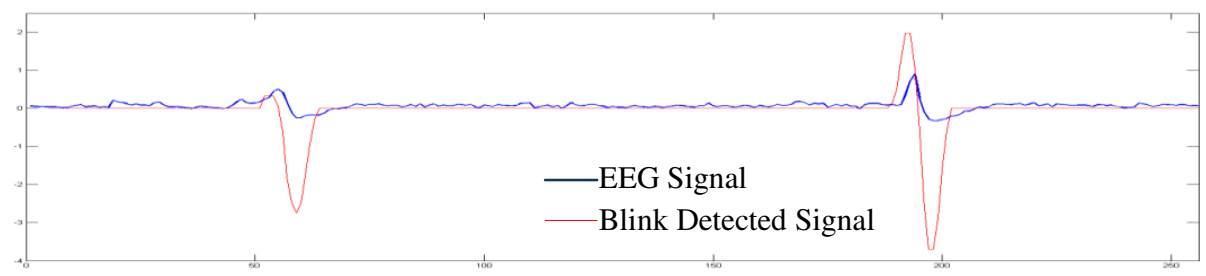

Fig. 9. Overlay of original EEG signal with the filtered EEG signal, highlighting the detected artifacts produced by eye blinking

\section{Results and Discussion}

Results from this work have been tested with real-time EEG introducing eye blinking artifacts alternately with different frequencies and amplitudes, proving to be a robust algorithm to filter artifacts produced by other sources that do not share similarity in shape, as well as detecting a target pattern in its many versions independent of the number of occurrences, the extent and the level of symmetry in the study window.
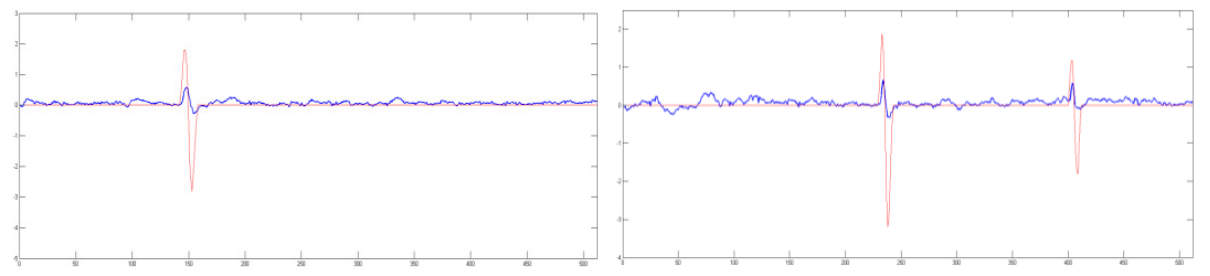

Fig. 10. Examples of real-time detection of a few eye blinks with an analysis window of $1000 \mathrm{~ms}$

As seen in Fig. 10 the pattern is always well-detected independent of the size of the analysis window. 


\section{Conclusions}

The use of wavelet transform in analyzing non-stationary signals, such as electroencephalograms, shows comparative advantages with respect to the Fourier transform. Among these advantages is the multi-resolution analysis which involves looking at the same time multiple versions of the same signal, the versions are band-pass filtered which are determined by the scale. Incorporating features of shape, amplitude, and phase shift for detecting patterns using one-dimensional wavelet transform ensures a robust recognition system prior to detection. This work contributes to the science related to bioengineering dedicated to brain computer interfaces or BCI, allowing real-time detection of artifacts or Eye Blinking; which generated voluntarily by the user can translate into simple commands for helping people with disabilities, for example, commanding the motor forward or reverse of a wheelchair. From the results of our work we conclude that filtering the artifact due to eye blinking in electroencephalogram EEG signals will be straightforward, now that the timing information of occurrence is well defined. It should be noted that although there are alternative analyses for eye blinking detection from EEG data, those approaches operate off-line, and our method operates in real time.

Acknowledgement. The authors gratefully acknowledge the support of DIMECUSACH and the support of VRID-USACH.

\section{References}

1. Gloor, P.: Hans Berger on the Electroencephalogram of Man. Elsevier Publishing Company, Amsterdam (1969)

2. Fisch, B.: EEG PRIMER Basic principles of digital and analog EEG, 3rd edn. Elsevier Academic Press (1999) ISBN: 0-444-82147-3

3. Binnie, C., Cooper, R., Mauguire, F., Osselton, J., Prior, P., Tedman, B.: Clinical Neurophysiology. Elsevier Academic Press (2003) ISBN: 0-444-51257-8

4. Polkko, J.: A Method for Detecting Eye Blinks from Single-Channel Biopotential Signal in the Intensive Care Unit. Master's Thesis (2007)

5. Slep, M.: Single Trial Analysis of EEG Signals, COS 497/498 (2003)

6. Bayliss, J.: A Flexible Brain-Computer Interface. Ph.D. Thesis, Computer Science Dept., U. Rochester (August 2001)

7. Chambayil, B., Singla, R., Jha, R.: EEG Eye Blink Classification Using Neural Network. In: Proceedings of the World Congress on Engineering, WCE 2010, London, U.K., vol. I (June 2010)

8. Walker, J.S.: Fourier Analysis and Wavelet Analysis. University of Wiconsin-Eau Claire. Notices of the Ams. 44(6) (1997)

9. Aeschbach, D., Borb'ely, A.A.: All-night dynamics of the human sleep EEG. J. Sleep Res. 2(2), 70-81 (1993)

10. Gilmore, R.L.: American Electroencephalographic Society guidelines in electroencephalography, evoked potentials, and polysomnography. J. Clin. Neurophysiol. 11, 147 (1994)

11. Sharbrough, F., Chatrian, G.-E., Lesser, R.P., Lüders, H., Nuwer, M., Picton, T.W.: American Electroencephalographic Society Guidelines for Standard Electrode Position Nomenclature. J. Clin. Neurophysiol. 8, 200-202 (1991) 
12. Divjak, M., Bischof, H.: Eye blink based fatigue detection for prevention of Computer Vision Syndrome. In: IAPR Conference on Machine Vision Applications (MVA 2009), Tokyo, Japan, May 20-22, pp. 350-353 (2009)

13. Mallat, S.: A theory for multiresolution signal decomposition: the wavelet repre-sentation. IEEE Pattern Anal. and Machine Intell. 11(7), 674-693 (1989)

14. Misiti, M., Misiti, Y., Oppenheim, G., Poggi, J.M.: Wavelet Toolbox for use with Matlab. The MathWorks Inc. EEUU (2004)

15. González, R., Woods, R.: Digital Image Processing, 3rd edn. Prentice-Hall (2008) 